\title{
Adalimumab combined with ciprofloxacin is superior to adalimumab monotherapy in perianal fistula closure in Crohn's disease: a randomised, double-blind, placebo controlled trial (ADAFI)
}

\author{
Pieter Dewint, ${ }^{1}$ Bettina E Hansen, ${ }^{1}$ Elke Verhey ${ }_{1}{ }^{1}$ Bas Oldenburg, ${ }^{2}$ \\ Daniel W Hommes, ${ }^{3}$ Marieke Pierik, ${ }^{4}$ Cyriel I J Ponsioen, ${ }^{5}$ Hendrik M van Dullemen, ${ }^{6}$ \\ Maurice Russel, ${ }^{7}$ Ad A van Bodegraven, ${ }^{8}$ C Janneke van der Woude ${ }^{1}$
}

For numbered affiliations see end of article.

Correspondence to Dr C Janneke van der Woude, Department of

Gastroenterology and Hepatology, Erasmus MC Rotterdam, 's Gravendijkwal 230, Rotterdam 3015 CE, The Netherlands; c.vanderwoude@erasmusmc.nl

Received 9 January 2013 Revised 22 February 2013 Accepted 22 February 2013 Published Online First 23 March 2013
To cite: Dewint $P$, Hansen $B E$, Verhey $E$, et al. Gut 2014;63:292-299.

\section{ABSTRACT}

Objective To assess whether a combination of adalimumab and ciprofloxacin is superior to adalimumab alone in the treatment of perianal fistulising Crohn's disease (CD).

Design Randomised, double-blind, placebo controlled trial in eight Dutch hospitals. In total, 76 CD patients with active perianal fistulising disease were enrolled. After adalimumab induction therapy (160/80 mg week 0, 2), patients received $40 \mathrm{mg}$ every other week together with ciprofloxacin $500 \mathrm{mg}$ or placebo twice daily for 12 weeks. After 12 weeks, adalimumab was continued. Follow-up was 24 weeks. Primary endpoint (clinical response) was defined as $50 \%$ reduction of fistulas from baseline to week 12. Secondary endpoints included remission (closure of all fistulas), Perianal Crohn's Disease Activity Index, Crohn's Disease Activity Index (CDAI) and Inflammatory Bowel Disease Questionnaire (IBDQ).

Results Clinical response was observed in $71 \%$ of patients treated with adalimumab plus ciprofloxacin and in $47 \%$ treated with adalimumab plus placebo ( $p=0.047$ ). Likewise, remission rate at week 12 was significantly higher $(p=0.009)$ in the combination group $(65 \%)$ compared with adalimumab plus placebo (33\%). Combination treatment was associated with a higher mean CDAl change and mean IBDQ change at week 12 ( $p=0.005$ and $p=0.009$, respectively). At week 24, no difference in clinical response between the two treatment groups was observed $(p=0.22)$. No difference in safety issues was observed. Conclusions Combination therapy of adalimumab and ciprofloxacin is more effective than adalimumab monotherapy to achieve fistula closure in CD. However, after discontinuation of antibiotic therapy, the beneficial effect of initial coadministration is not maintained.

Trial registration ClinicalTrials.gov Identifier: NCT00736983.

\section{BACKGROUND}

Perianal fistulising disease occurs in up to $40 \%$ of Crohn's disease (CD) patients and its natural history is characterised by chronicity with only rarely spontaneous healing of the fistula tracts. ${ }^{1}{ }^{2}$ Perianal problems have a negative impact on the perceived health related quality of life $^{3}$ and despite intensive medical and surgical treatment, a considerable percentage of patients will continue to have debilitating perianal

\section{Significance of this study}

What is already known on this subject?

- Perianal fistulas are common in patients with Crohn's disease and negatively impact quality of life.

- Spontaneous healing of fistula tracts seldom occurs.

- Antitumour necrosis factor antibodies are effective for the treatment of Crohn's disease related fistulas.

What are the new findings?

- Combination therapy of adalimumab and ciprofloxacin results in a higher fistula closure rate and results in a better quality of life than adalimumab therapy alone.

- After discontinuation of ciprofloxacin the initial beneficial effect on fistula closure is not sustained.

- Previous administration of infliximab does not influence the effect of adalimumab to induce fistula closure.

How might it impact on clinical practice in the foreseeable future?

- To induce fistula closure in patients with perianal fistulising Crohn's disease the association of ciprofloxacin with adalimumab is preferred over adalimumab alone.

symptoms. This ultimately leads to extensive surgery such as proctocolectomy. ${ }^{2}$ Antibiotics are used for fistulising $\mathrm{CD}$ and this is primarily based on uncontrolled data, demonstrating response with metronidazole in up to $83 \%$ of patients. ${ }^{5}$ A randomised trial comparing metronidazole, ciprofloxacin and placebo failed to show the superiority of antibiotic treatment, largely ascribed to the relatively small sample size. ${ }^{6}$ Infliximab (antitumour necrosis factor (anti-TNF)) demonstrated to induce and maintain response in patients with fistulising $\mathrm{CD} .^{78}$ The CHARM trial, a randomised, placebo controlled trial investigating the role of adalimumab, a fully humanised monoclonal anti-TNF antibody, in CD, and in which the 
closure of fistulas was a secondary endpoint, documented that adalimumab induced a reduction in the mean number of draining fistulas per day compared with placebo during a treatment period of 1 year. $^{9}$ In the open-label extension study of the CHARM, 90\% of patients maintained response to fistula treatment after an additional year of treatment with adalimumab. ${ }^{10}$

We previously demonstrated that combination therapy of infliximab and ciprofloxacin was associated with a trend to higher response compared with infliximab alone, suggesting that anti-TNF treatment for fistulising CD combined with ciprofloxacin is more effective than anti-TNF alone. ${ }^{11}$ Here, we report the ADAFI trial (ADAlimumab for the treatment of perianal FIstulas in Crohn's disease) investigating combination therapy of adalimumab with ciprofloxacin or placebo in a randomised, controlled fashion for the treatment of perianal fistulising CD.

\section{METHODS}

\section{Study design and sample size}

This multicentre, randomised, double-blind, placebo controlled trial was designed to evaluate the efficacy and safety of combination therapy of adalimumab and ciprofloxacin (ADA+CIP) in comparison with adalimumab and placebo (ADA+PLA) for the treatment of $\mathrm{CD}$ related, active perianal fistulising disease (ClinicalTrials.gov number NCT00736983). The ADAFI trial was conducted in accordance with the protocol International Conference on Harmonization Guidelines for Good Clinical Practice, the Declaration of Helsinki and local national regulations governing clinical study conduct. The protocol was approved by the institutional review board or by the ethics committee at each centre. The study was not designed for an interim analysis and no Data Safety Monitoring Board was assigned. All patients gave written informed consent. Patients were enrolled at eight sites in The Netherlands from September 2008 to March 2011. The sample size calculation was based on results from a small open-label study, ${ }^{12}$ at the time of the study design presented in abstract form (Digestive Disease Week 2006), reporting that adalimumab administration resulted in $64 \%$ of patients in closure of at least $50 \%$ of the number of draining fistulas. An absolute increment of $20 \%$ of patients reaching the primary endpoint in the combination treatment arm was deemed to be clinically relevant. Using the two-group $\chi^{2}$ test for equal proportions and equal group size and with the power fixed to $80 \%$ and a two-sided significance level of $\alpha=0.05$, the sample size was calculated to be 73 patients per group.

\section{Intervention and randomisation}

All patients received an induction regimen with $160 \mathrm{mg}$ adalimumab at day 0 and $80 \mathrm{mg}$ at week 2. At week 4, patients received $40 \mathrm{mg}$ adalimumab every other week until the end of follow-up at week 24. Patients self-administered adalimumab by subcutaneous injection as prescribed by the manufacturer. Patients were randomly assigned to additionally receive ciprofloxacin $500 \mathrm{mg}$ or placebo twice daily from day 0 on for 12 weeks. Randomisation was performed through a centralised randomisation schedule in a 1:1 ratio. Perianal setons, if present, were removed at week 2 .

\section{Patients}

Men and women diagnosed with CD and between 18 and 70 years of age with active fistulising perianal disease at screening and baseline were included. Previous treatment with infliximab was permitted if infliximab had been discontinued at least 12 weeks before the screening visit and the patient had initially experienced response to the agent (as judged by the investigating physician). Baseline fistula data were noted.
Demographic and baseline CD severity data, concomitant medication use, previous history of TNF-antagonist use and smoking history were collected. The diagnosis of CD (at least 3 months in duration) was radiologically or endoscopically/ histologically confirmed. Women of childbearing potential were required to use an effective form of birth control. Concomitant use of thiopurine derivatives, methotrexate and 5 -aminosalicylic acid was allowed provided the dose was stable for at least 12 weeks. Oral corticosteroids were allowed if patients initiated this treatment at least 4 weeks prior to randomisation and if they were on a stable dose not above $20 \mathrm{mg} /$ day of prednisone equivalent over the 2 weeks prior to randomisation. From 6 weeks after randomisation of a subject, it was advocated to taper corticosteroid treatment. Use of antibiotics for CD within 3 months prior to randomisation and a history of allergy to quinolones or previous treatment with adalimumab were exclusion criteria. Patients were also excluded if they had a history of lymphoproliferative disease or cancer, other than skin basocellular carcinoma; listeriosis; HIV infection; immunodeficiency syndrome; central nervous system demyelinating disease; chronic hepatitis $\mathrm{B}$ or $\mathrm{C}$ virus infection; or untreated tuberculosis at baseline. Patients were excluded if they had perianal abscesses; renal insufficiency (elevated serum creatine), significant pulmonary dysfunction, poorly controlled medical conditions, including diabetes mellitus and unstable ischaemic heart disease; a history of QT prolongation; a known pre-existing condition that could interfere with the patient's participation such as psychiatric conditions, central nervous system trauma or active seizure disorders. In addition, patients were excluded if they had undergone treatment with any investigational agent in the past 12 weeks or five half-lives prior to the screening visit. Patients with a history of clinically significant drug or alcohol abuse in the last 2 years were not allowed to participate in this study.

\section{Study endpoints}

At baseline, week 12 and week 24, a dedicated, clinical examination of rectum and perineum with gentle compression of fistula tracts was performed by a physician blinded for the treatment allocation to assess the number of draining fistulas and to exclude the presence of abscesses. Patients who did not have draining fistulas at the screening or baseline visits were excluded from this trial. The primary outcome of the ADAFI was at least $50 \%$ reduction of the number of draining fistulas from baseline to week 12. The proportion of patients with closure of all draining fistulas from baseline, defined as remission, at week 12 and week 24 was a secondary outcome.

The Perianal Disease Activity Index (PDAI), ${ }^{13}$ Crohn's Disease Activity Index (CDAI) and Inflammatory Bowel Disease Questionnaire (IBDQ) ${ }^{14}$ were obtained both at baseline, as well as at week 12 and week 24. The PDAI consists of five items, each item graded on a 5-point Likert scale with total PDAI score ranging from 0 to 20 . The CDAI scores range from 0 to 600 , with higher scores indicating more severe disease activity. The IBDQ is a health related quality of life questionnaire, with a total score which ranges from 32 to 224 and is derived from scores in four domains: bowel (10 items), systemic (five items), emotional (12 items) and social (five items). ${ }^{15}$

Serious adverse events and other adverse events of interest were collected throughout the study. An opportunistic infection was defined as either an infection due to an organism which usually does not cause disease or an unusually severe infection by an organism that normally causes mild disease only. 


\section{Data and statistical analysis}

All analyses were performed using the modified intention to treat (M-ITT) principle in the cohort of randomised participants who fulfilled the inclusion criteria, gave written informed consent and in whom setons were removed according to protocol. Additional per protocol (PP) analyses were performed.

For the primary endpoint, patients not evaluated at the scheduled time points were coded as non-responders. At week 12, a sample size of 70 evaluable study participants for M-ITT was available. Categorical variables were described globally or per treatment group using frequencies and percentages. Their distributions were compared between treatment groups by using the $\chi^{2}$ test or the Fisher exact test when necessary. Continuous variables were summarised using the median and IQR or the mean and SD and their distributions were compared between treatment groups using the Mann-Whitney test.

Frequencies of response, adverse events at weeks 12 and 24 were compared between treatment groups by using the $\chi^{2}$ test or the Fisher exact test.
Results were considered significant when the $\mathrm{p}$ value was less than 0.05 . An $\alpha$-level of 0.01 was applied for IBDQ subscales to control for multiple testing. Data were analysed with SPSS Software for Windows, release V.17 (SPSS Inc, Chicago, Illinois, USA).

\section{RESULTS}

\section{Patient flow}

Enrolment of participants required more time than anticipated. Therefore, the initial enrolment period fixed to 12 months was twice extended to ultimately 30 months in an effort to include the required sample size. Further prolongation of inclusion period was financially not feasible especially due to the costs related to production of blinded medication and patient insurance, necessitating closure of the study before reaching the calculated number of patients. A total of 76 patients were enrolled in the study. In all, 37 patients were allocated to ADA+CIP and 39 patients to ADA+PLA treatment (figure 1). Six patients, three from each group, were excluded from analysis due to the following reasons: four had no draining fistula at baseline and in two patients the drain was not removed according to the protocol.

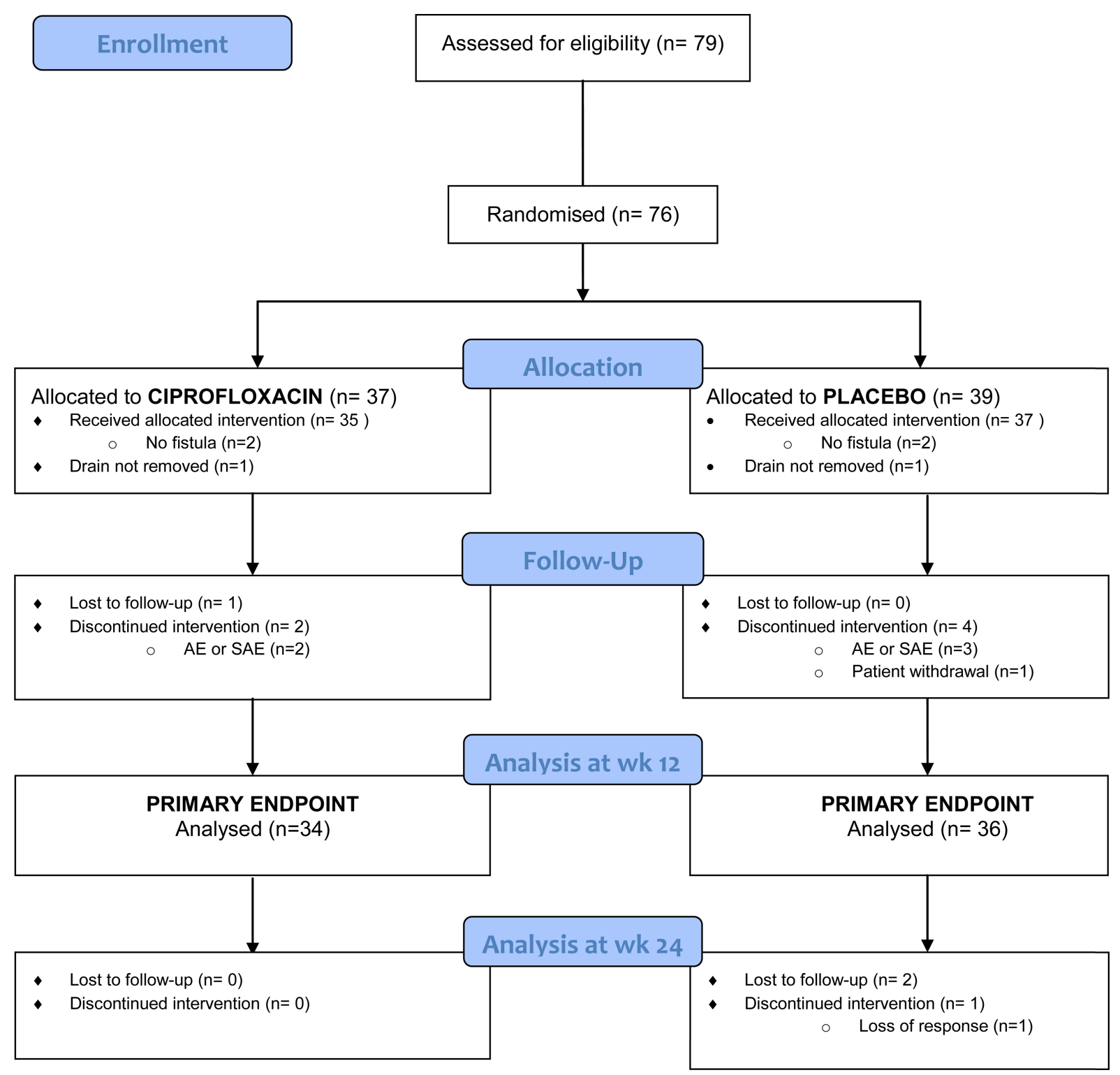

Figure 1 Study flow chart. 


\section{Baseline characteristics}

Table 1 shows demographic and clinical characteristics by treatment in the ADA+CIP $(n=34)$ or ADA+PLA $(n=36)$ group. Baseline characteristics were similar between the two groups, indicative of successful randomisation. The mean age of the patients was 36.1 years, $52.9 \%$ were male subjects and $15.7 \%$ had a stoma in situ. In total, 24 (34\%) patients were previously

Table 1 Patient baseline demographics and clinical characteristics

\begin{tabular}{|c|c|c|}
\hline & Ciprofloxacin & Placebo \\
\hline \multicolumn{3}{|l|}{ Baseline randomised } \\
\hline $\mathrm{N}$ & 34 & 36 \\
\hline \multicolumn{3}{|l|}{ Age } \\
\hline Mean (SD) & $34.7(11.0)$ & $37.3(12.4)$ \\
\hline Median (min-max) & $31(19-63)$ & $37(18-68)$ \\
\hline \multicolumn{3}{|l|}{ Sex } \\
\hline Male & $20(58.8 \%)$ & $17(47.2 \%)$ \\
\hline Female & $14(41.2 \%)$ & $19(52.8 \%)$ \\
\hline \multicolumn{3}{|l|}{ Race } \\
\hline Caucasian & $31(91.2 \%)$ & $33(91.7 \%)$ \\
\hline Asian & $1(2.9 \%)$ & $1(2.8 \%)$ \\
\hline African & 0 & $2(5.6 \%)$ \\
\hline Other & $2(5.9 \%)$ & 0 \\
\hline \multicolumn{3}{|l|}{ Smoking } \\
\hline $\mathrm{N}(\%)$ & $12(35.3 \%)$ & $10(27.8 \%)$ \\
\hline \multicolumn{3}{|l|}{ Stoma } \\
\hline N (\%) & $3(8.8 \%)$ & $8(22.2 \%)$ \\
\hline \multicolumn{3}{|l|}{ Previous infliximab } \\
\hline $\mathrm{N}(\%)$ & $12(35.2 \%)$ & $12(33.3 \%)$ \\
\hline Concomitant medication $\mathrm{N}(\%)$ & $14(41.2 \%)$ & $13(36.1 \%)$ \\
\hline Prednisolon & $3(8.8 \%)$ & $1(2.8 \%)$ \\
\hline Any immunosuppressant & $13(38.2 \%)$ & $12(33.3 \%)$ \\
\hline Azathioprine & $9(26.5 \%)$ & $9(25 \%)$ \\
\hline 6-Mercaptopurine & $3(8.8 \%)$ & $1(2.8 \%)$ \\
\hline Methotrexate & $1(2.9 \%)$ & $2(5.6 \%)$ \\
\hline \multicolumn{3}{|l|}{ Number of draining fistulas } \\
\hline Mean (SD) & $1.59(1.02)$ & $1.56(1.00)$ \\
\hline Median (min-max) & $1(1-5)$ & $1(1-6)$ \\
\hline 1 & 22 & 23 \\
\hline 2 & 8 & 9 \\
\hline 3 & 1 & 3 \\
\hline$\geq 4$ & 3 & 1 \\
\hline \multicolumn{3}{|l|}{ Seton at baseline } \\
\hline $\mathrm{N}(\%)$ & $8(23.5 \%)$ & $7(19.4 \%)$ \\
\hline \multicolumn{3}{|l|}{ Number of setons } \\
\hline 1 & $4(11.8 \%)$ & $3(8.3 \%)$ \\
\hline$>1$ & $4(11.8 \%)$ & $4(11.1 \%)$ \\
\hline \multicolumn{3}{|l|}{ PDAI score } \\
\hline Mean (SD) & $7.59(3.26)$ & $7.47(2.83)$ \\
\hline Median (min-max) & $7.5(2-17)$ & $7(2-13)$ \\
\hline \multicolumn{3}{|l|}{$\mathrm{CDAl}^{*}$} \\
\hline Mean (SD) & $173.2(96.7)$ & $136.5(67.1)$ \\
\hline Median (min-max) & $150.5(32-381)$ & $118(15-287)$ \\
\hline \multicolumn{3}{|l|}{ IBDQ } \\
\hline Mean (SD) & $157.6(34.9)$ & $162.9(30.0)$ \\
\hline Median (min-max) & $160(81-213)$ & $168(95-209)$ \\
\hline
\end{tabular}

exposed to infliximab. Approximately $40 \%$ of the patients were treated with concomitant CD related medications during the study. At baseline the mean number of draining fistula was 1.57 (SD 1.0) with a mean PDAI score of 7.53 (SD 3.03). The mean CDAI was 155.8 (SD 85.3) indicating a mildly active (luminal) disease with a mean IBDQ score of 160 , consistent with a low quality of life in patients with fistulising CD.

\section{Outcomes}

Table 2 shows the main endpoints of study. At week 12, ADA + CIP was associated with a significant higher number of patients with a closure of at least $50 \%$ of draining fistulas compared with patients randomised to ADA+PLA (M-ITT: 71\% vs 47\%, p=0.047; PP: $75 \%$ vs $51.5 \%, p=0.0498$ ) (figure 2). Univariate analysis excluded that baseline patient characteristics influenced fistula closure in patients receiving ciprofloxacin or placebo (table 3). In a post hoc logistic regression analysis of the treatment effect, we adjusted for a possible effect of the presence or non-presence of stoma, and the treatment effect stayed significant $(p=0.025)$. Similarly for baseline CDAI and presence of setons at baseline, the treatment effect stayed significant $(\mathrm{p}=0.045$ and 0.046$)$.

Remission was observed in 22 out of 34 patients $(65 \%)$ on $\mathrm{ADA}+\mathrm{CIP}$ at week 12, whereas this was observed in only 12 of $36(33 \%)$ patients on ADA+PLA $(p=0.009)$ in an M-ITT population. In the PP population, the $\mathrm{p}$ value remained highly significant $(p=0.009)$ with remission in $69 \%$ of patients on ADA + CIP versus $36 \%$ on ADA+PLA.

At week 24 (12 weeks after stopping the ciprofloxacin), the difference in fistula closure rates between the two treatment groups was not maintained; in the M-ITT population, 21 of 34 patients $(62 \%)$ and 17 of 36 patients (47\%) in ADA+CIP and ADA+PLA groups, respectively, had at least $50 \%$ reduction of the number of draining fistula $(p=0.22)$ (figure 2). Complete response was observed in 18 of 34 (53\%) and 12 of 36 (33\%) of patients in ADA+CIP and ADA+PLA, respectively $(p=0.098)$. Similar, non-significant differences between the two groups were observed in the PP population at week 24 for both $50 \%$ and $100 \%$ reduction of number of draining fistula (data not shown).

The median PDAI at week 12 decreased $(\mathrm{p}<0.001)$ compared with baseline in both treatment arms. In ADA+CIP, the PDAI decreased from $7.5(2-17)$ at baseline to $2.0(0-16)$ at week 12, mirroring the decrease in PDAI observed in the ADA+PLA, from $7(2-13)$ to $4(0-10)$ at week 12. At week 24, median PDAI was $2(0-17)$ in ADA+CIP and $3.5(0-16)$ in ADA+PLA. A statistically significant difference between the two treatment arms was observed neither at week 12 nor at week 24 .

For the CDAI analysis, patients with a stoma were excluded for analysis. At week 12, patients treated with ADA+CIP experienced a significantly higher decrease in mean CDAI compared with ADA+PLA $(p=0.005)$. At week 24 , this difference remained statistically significant $(p=0.005)$. This difference in CDAI change at week 12 and week 24 could not be attributed to a specific decrease of one of its constituting domains of variables (data not shown).

At week 12, the mean IBDQ change in ADA+CIP was 23.2 versus 10.4 in ADA+PLA $(p=0.009)$. At week 24 , no difference $(p=0.62)$ between the two treatment arms could be observed with a mean IBDQ change of 16.5 versus 12.7 in ADA+CIP and ADA+PLA, respectively. Subdividing the IBDQ in its subscales and after correcting for multiple testing, a significant difference in mean IBDQ subscale score change was observed at week 12 for the domains of bowel symptoms and of social 
Table 2 Main endpoints

\begin{tabular}{|c|c|c|c|c|c|c|}
\hline & \multicolumn{3}{|l|}{ Week 12} & \multicolumn{3}{|l|}{ Week 24} \\
\hline & $\begin{array}{l}\text { Ciprofloxacin } \\
n=34\end{array}$ & $\begin{array}{l}\text { Placebo } \\
n=36\end{array}$ & $\mathrm{p}$ Value & $\begin{array}{l}\text { Ciprofloxacin } \\
n=34\end{array}$ & $\begin{array}{l}\text { Placebo } \\
n=36\end{array}$ & p Value \\
\hline \multicolumn{7}{|c|}{$50 \%$ reduction in number of fistulas } \\
\hline $\mathrm{n}$ & 24 & 17 & & 21 & 17 & \\
\hline$\%(95 \% \mathrm{Cl})$ & $71 \%$ (54 to 87$)$ & $47 \%$ (30 to 64$)$ & 0.047 & $62 \%$ (45 to 79 ) & $47 \%$ (30 to 64$)$ & 0.22 \\
\hline \multicolumn{7}{|c|}{$100 \%$ reduction in number of fistulas } \\
\hline $\mathrm{n}$ & 22 & 12 & & 18 & 12 & \\
\hline$\%(95 \% \mathrm{Cl})$ & $65 \%$ (48 to 82 ) & $33 \%(17$ to 50$)$ & 0.009 & $53 \%$ (35 to 71$)$ & $33 \%(17$ to 50$)$ & 0.098 \\
\hline \multicolumn{7}{|l|}{ PDAl, median (range) } \\
\hline Score & $2(0-16)$ & $4(0-10)$ & & $2(0-17)$ & $3.5(0-16)$ & \\
\hline$\Delta$ Compared with week 0 & $5(-1-9)$ & $3(-1-10)$ & 0.16 & $4(-1-9)$ & $3(-6-12)$ & 0.32 \\
\hline \multicolumn{7}{|l|}{ PDAI, median (IQR) } \\
\hline Score & $2(1-5)$ & $4(2-5)$ & & $2(0-17)$ & $3.5(0-16)$ & \\
\hline$\Delta$ Compared with week 0 & $5(3.0-6.0)$ & $3(1.25-5.75)$ & 0.16 & $4(2-7)$ & $3(1-6.5)$ & 0.32 \\
\hline CDAl, mean $(95 \% \mathrm{Cl})$ & $\mathrm{n}=28$ & $\mathrm{n}=25$ & & $\mathrm{n}=25$ & $\mathrm{n}=22$ & \\
\hline Score & 105 (74 to 136$)$ & 113 (82 to 145 ) & & $120(78$ to 161$)$ & 122 (79 to 166$)$ & \\
\hline$\Delta$ Compared with week 0 & 74 (48 to 101) & $23(6$ to 40$)$ & 0.002 & 67 (30 to 104) & $8(-18$ to 33$)$ & 0.01 \\
\hline IBDQ, mean $(95 \% \mathrm{CI})$ & $\mathrm{n}=30$ & $n=30$ & & $n=30$ & $\mathrm{n}=27$ & \\
\hline Score & 181 (172 to 191$)$ & 173 (161 to 186$)$ & & 174 (160 to 189$)$ & 178 (166 to 191) & \\
\hline$\Delta$ Compared with week 0 & 23 (16 to 30$)$ & 10 (4 to 17$)$ & 0.009 & 17 (6 to 27$)$ & 13 (1 to 25$)$ & 0.69 \\
\hline \multicolumn{7}{|l|}{ IBDQ bowel, mean (95\% Cl) } \\
\hline Score & $57(55$ to 60$)$ & 54 (51 to 57$)$ & & 53 (49 to 58$)$ & 56 (53 to 59$)$ & \\
\hline$\Delta$ Compared with week 0 & 9 (6 to 12$)$ & $4(2$ to 6$)$ & 0.007 & 5 (1 to 9$)$ & 6 (2 to 9$)$ & 0.77 \\
\hline \multicolumn{7}{|l|}{ IBDQ systemic, mean (95\% CI) } \\
\hline Score & 25 (23 to 27$)$ & 24 (22 to 27$)$ & & 25 (22 to 27$)$ & 26 (23 to 29$)$ & \\
\hline$\Delta$ Compared with week 0 & $4(3$ to 6$)$ & $2(0$ to 4$)$ & 0.03 & $4(1$ to 6$)$ & $3(0$ to 6$)$ & 0.71 \\
\hline \multicolumn{7}{|l|}{ IBDQ emotion, mean $(95 \% \mathrm{Cl})$} \\
\hline Score & 68 (64 to 73 ) & 65 (60 to 71$)$ & & 67 (61 to 73$)$ & 66 (60 to 72 ) & \\
\hline$\Delta$ Compared with week 0 & 5 (2 to 8$)$ & $3(0$ to 7$)$ & 0.45 & $4(0$ to 7$)$ & $3(-2$ to 9$)$ & 0.9 \\
\hline \multicolumn{7}{|l|}{ IBDQ social, mean (95\% Cl) } \\
\hline Score & 30 (38 to 32 ) & 30 (27 to 32 ) & & 29 (26 to 32 ) & 30 (27 to 32 ) & \\
\hline$\Delta$ Compared with week 0 & 5 (3 to 7$)$ & 1 (0 to 3 ) & 0.002 & $4(1$ to 7$)$ & $1(-1$ to 4$)$ & 0.12 \\
\hline
\end{tabular}

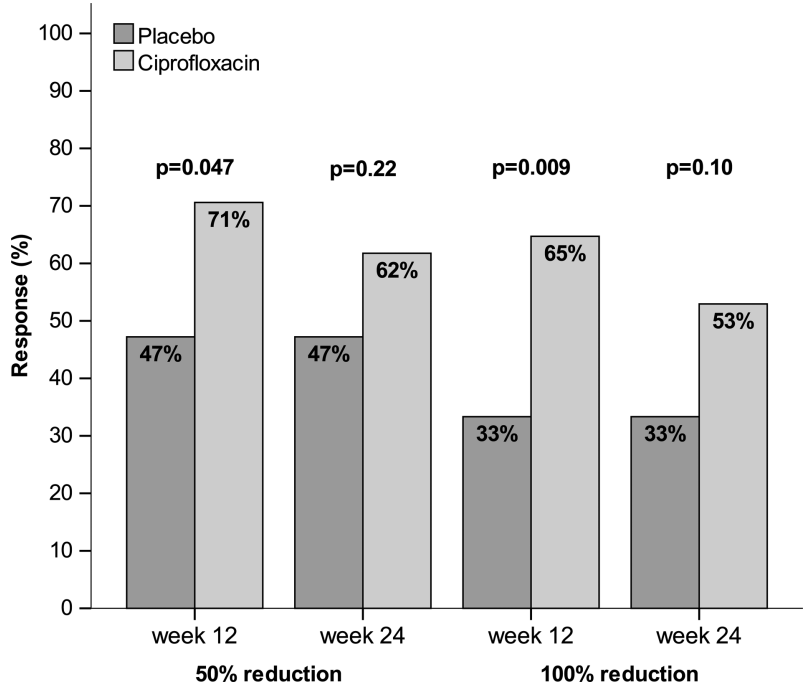

Figure 2 Percentage of patients with response $(\geq 50 \%$ closure of draining fistulas from baseline) and remission (100\% closure of draining fistulas) at week 12 (primary endpoint) and at week 24 in the two treatment arms. symptoms ( $p=0.007$ and $\mathrm{p}=0.002$, respectively) in the ADA + CIP compared with the ADA+PLA group.

\section{Adverse events}

The adverse event rates were similar regarding frequency or severity of adverse events observed between the two groups (see table 4). Serious adverse events were reported in five patients, observed in three patients in the ADA+CIP and in two patients in the ADA+PLA treatment arm. These serious adverse events included one case of systemic herpes simplex infection in the ADA+PLA arm occurring 2 weeks after the first adalimumab dose and one case of sagittal sinus thrombosis in the ADA+CIP arm, not considered to be associated with therapy. The other adverse events scored as serious adverse event were two exacerbations of CD (ADA + CIP treatment arm) and one patient hospitalised for acute pain due to parastomal herniation, which spontaneously resolved within $24 \mathrm{~h}$ (ADA+PLA treatment arm). In total, 91.2\% (31/34) and 94.4\% (34/36) $(\mathrm{p}=0.60)$ of patients in ADA+CIP and ADA+PLA arms, respectively, experienced adverse events. The most frequently occurring adverse event was infection: 19 out of $34(55.9 \%)$ in ADA+CIP compared with 22 out of $36(61.1 \%)$ of the patients in ADA+PLA experienced an infectious adverse event. The majority of these constituted of upper respiratory tract infections $(50.0 \%$ and 
Table 3 Univariate analysis

\begin{tabular}{|c|c|c|c|}
\hline & \multicolumn{2}{|c|}{ Primary endpoint } & \multirow[b]{2}{*}{ p Value } \\
\hline & Yes & No & \\
\hline \multicolumn{4}{|l|}{ Sex } \\
\hline Male & $21(56.8 \%)$ & $16(43.2 \%)$ & 0.74 \\
\hline Female & $20(60.6 \%)$ & $13(39.4 \%)$ & \\
\hline \multicolumn{4}{|l|}{ Race } \\
\hline Caucasian & 36 (56.3\%) & $28(43.8 \%)$ & 0.39 \\
\hline Other & $5(83.3 \%)$ & $1(16.7 \%)$ & \\
\hline \multicolumn{4}{|l|}{ Seton } \\
\hline Yes & $9(60 \%)$ & $6(40 \%)$ & 0.90 \\
\hline No & $32(58.2 \%)$ & $23(41.8 \%)$ & \\
\hline \multicolumn{4}{|l|}{ Stoma } \\
\hline Yes & $8(72.7 \%)$ & $3(27.3 \%)$ & 0.30 \\
\hline No & $33(55.9 \%)$ & $26(44.1 \%)$ & \\
\hline \multicolumn{4}{|l|}{ Tobacco } \\
\hline Yes & $12(54.5 \%)$ & $10(45.5 \%)$ & 0.64 \\
\hline No & $29(60.4 \%)$ & $19(39.6 \%)$ & \\
\hline \multicolumn{4}{|l|}{ Previous IFX } \\
\hline Yes & $15(62.5 \%)$ & $9(37.5 \%)$ & 0.63 \\
\hline No & $26(56.5 \%)$ & $20(43.5 \%)$ & \\
\hline \multicolumn{4}{|c|}{ Corticosteroids } \\
\hline Yes & $2(50 \%)$ & $2(50 \%)$ & 0.72 \\
\hline No & $39(59.1 \%)$ & $27(40.9 \%)$ & \\
\hline \multicolumn{4}{|c|}{ AZA/6MP/MTX } \\
\hline Yes & $12(48.0 \%)$ & $13(52.0 \%)$ & 0.18 \\
\hline No & 29 (46.4\%) & $16(35.6 \%)$ & \\
\hline
\end{tabular}

$55.6 \%$ of patients, respectively). Opportunistic infections were reported in 5/34 (14.7\%) in ADA+CIP versus 4/36 (11.1\%) patients treated with ADA+PLA. All of these opportunistic infections but one were mild adverse events, including skin related ailments like vaginal candidiasis or onychomycosis.

Table 4 Summary of safety and occurrence of adverse events

\begin{tabular}{lccc}
\hline & $\begin{array}{l}\text { Ciprofloxacin } \\
(\mathrm{N}=34)\end{array}$ & $\begin{array}{l}\text { Placebo } \\
(\mathbf{N}=36)\end{array}$ & p Value \\
\hline Serious AE & $3(8.8 \%)$ & $3(8.3 \%)$ & 1.00 \\
Any AE & $31(91.2 \%)$ & $34(94.4 \%)$ & 0.60 \\
Most common AE & & & \\
$\quad$ URTI & $17(50.0 \%)$ & $20(55.6 \%)$ & 0.64 \\
Fatigue & $10(29.4 \%)$ & $12(33.3 \%)$ & 0.72 \\
$\quad$ Headache & $10(29.4 \%)$ & $6(16.7 \%)$ & 0.20 \\
$\quad$ Nausea & $7(20.6 \%)$ & $3(8.3 \%)$ & 0.18 \\
Infection & $19(55.9 \%)$ & $22(61.1 \%)$ & 0.66 \\
$\quad$ Serious infectious AE & $0(0 \%)$ & $1(2.8 \%)^{*}$ & 1.00 \\
$\quad$ Opportunistic infection & $5(14.7 \%) \dagger$ & $4(11.1 \%) \ddagger$ & 0.73 \\
$\quad$ Perianal abscess & $1(2.9 \%)$ & $2(5.6 \%)$ & 1.00 \\
Thromboembolic AE & $1(2.9 \%) \S$ & 0 & 0.49 \\
Worsening Crohn's disease & $3(8.8 \%)$ & $3(8.3 \%)$ & 1.00 \\
\hline tSstic
\end{tabular}

*Systemic herpes simplex infection.

‡Four events in four patients: two events of mucocutaneous mycosal infection, one event of systemic herpes infection, one event of HPV related warts.

tSeven events in five patients: five mucocutaneous mycosal infections, one event of herpes labialis, one event of enlargement of a pre-existing genital human papillomavirus wart.

$\S$ Sagittal sinus thrombosis.

$A E$, adverse event; URTI, upper respiratory tract infection.
The occurrence of three perianal abscess was documented: one in the ADA+CIP treatment arm and two in the ADA+PLA treatment.

\section{DISCUSSION}

This is the first trial demonstrating a superior effect of combination therapy of adalimumab with ciprofloxacin compared with adalimumab monotherapy to achieve fistula closure in CD related perianal fistulising disease. A placebo controlled study, evaluating combination therapy with infliximab and ciprofloxacin versus infliximab alone was unable to show a difference in response rate of perianal fistulas in $\mathrm{CD}$, ascribed to the small sample size. ${ }^{11}$ Interestingly, although a clear trend to a higher remission rate in patients treated with combination therapy is observed at week 24, no significant difference for the primary endpoint was observed 12 weeks after discontinuation of ciprofloxacin. This was solely due to loss of response in three patients randomised to the combination treatment group, whereas the number of patients in the adalimumab monotherapy group who had reached the primary endpoint at week 12 remained stable at week 24, which is consistent with data from the CHARM trial, demonstrating long term maintenance of fistula closure. ${ }^{10}$

Univariate analysis showed that outcome was not influenced by any of the patient characteristics. Although, due to the small number of patients in each subgroup, caution should be exercised in further interpreting this analysis, it is interesting to note that previous treatment with infliximab did not impair fistula closure with adalimumab. This is in agreement with data from the CHOICE trial, an open-label study evaluating efficacy in CD patients failing on infliximab treatment, in which complete fistula closure was observed in $39 \%$ of patients. ${ }^{16}$ Because of the (not significant) imbalance between the two groups regarding the presence of stoma and baseline CDAI, a post hoc analysis was performed. The treatment effect after correction for these factors remained significant.

Although the overall, mean CDAI at baseline reflected an almost quiescent luminal disease status of the randomised patients, the reduction of mean CDAI at week 12 was statistically significantly larger in the combination treatment group compared with adalimumab alone. This is in line with results from a randomised trial showing effectiveness of ciprofloxacin in the treatment in moderately active $\mathrm{CD} .{ }^{17} \mathrm{~A}$ recently published cohort study, ${ }^{18}$ evaluating the use of autologous bone derived mesenchymal stromal cells in the treatment of fistulising CD after administration of broad-spectrum antibiotics for 2 weeks immediately prior to the first injection procedure, also demonstrated a significant decrease of CDAI after treatment. However, this was largely due to an increase of general well-being, which is one of the eight variables constituting the CDAI. In our patient group, however, the decrease of CDAI could not be attributed to selective decrease of one of its subdomains.

It has been shown that patients in symptomatic remission usually have an IBDQ of at least $170 .{ }^{19}{ }^{20}$ Although the mean CDAI in our patient group was only slightly higher than 150 , the mean IBDQ at baseline was noticeably below 170 points, which suggests that particularly perianal fistulas in CD contribute negatively to quality of life. To corroborate this observation, 12 weeks of combination therapy with adalimumab and ciprofloxacin resulted in a more marked increase of IBDQ compared with adalimumab monotherapy, with an absolute increase of 23 points, being defined as clinically relevant. ${ }^{20}$

Although the number of patients included in this trial is too small to draw firm conclusions with respect to adverse events associated with combination therapy, it is reassuring that the 
rate of adverse events in the combination therapy group is not increased. This might especially be of importance regarding the occurrence of non-bacterial opportunistic infections, which might be associated with use of antibiotics. Additionally, infections with Clostridium difficile were not observed. High rates of perianal abscess formation have been described after treatment with anti-tumour necrosis factor (TNF) and it is therefore advised to perform additional imaging (magnetic resonance imaging (MRI) or endo-anal ultrasound (EUS)) prior to start of anti-TNF therapy and to insert drains in perianal fistula tracts to optimise drainage and prevent the premature closure of the fistula. Interestingly, in this study abscess related adverse events were low, even in the absence of perianal drains at baseline or without prior MRI, suggesting that a well-executed clinical examination might be equivalent in the detection of insufficient drainage. Moreover, no difference in abscess related adverse events was detected between combination and adalimumab monotherapy, arguing against a role for ciprofloxacin in the prevention of abscesses. However, a possible significant difference might have been obscured by the low number of abscesses observed in the adalimumab alone group.

Although the inclusion period was prolonged with 16 months in an attempt to reach the calculated sample size, fewer patients than anticipated were included. Although the overestimation of beneficial effect is a well-recognised problem in randomised controlled trials stopped early for overt efficacy, ${ }^{21}$ the situation for trials stopped for economic reasons is more elusive. As this trial was stopped solely for economical reasons, without performing an interim analysis, the results in this randomised controlled trial are unlikely to be attributed to a possible underpowered study. Moreover, the result of a small randomised controlled trial comparing combination therapy of infliximab and ciprofloxacin with infliximab monotherapy was already suggestive for a benefit for combination therapy, ${ }^{11}$ supporting our current findings. As the design of both studies is comparable, a meta-analysis of the effect of antibiotics on top of anti-TNF treatment would be of high interest.

In this study, imaging with MRI or EUS was not performed at baseline nor at a later time point and the assessment of perianal fistulas or abscesses was solely based on dedicated clinical investigation. As it has been described that clinical judgment of fistula closure might not correlate with true fistula healing as evaluated with ultrasound and MRI, ${ }^{22-24}$ this might result in an overestimation of the treatment effect. However, a recent study described that the median lag between radiological healing and clinical remission is 12 months, thereby underestimating the benefit of anti-TNF therapy in the short term. Moreover, after stopping anti-TNF treatment, the recurrence of draining fistulas was observed even by radiology determined healed fistula tracts, questioning the additive clinical value of MRI to clinical investigation. $^{25}$ The same conclusion can be drawn from the study of Karmiris et $a l^{26}$ describing that MRI underestimates the clinical response to long term anti-TNF, thereby arguing against sufficient specificity of MRI to detect fistula healing in long term follow-up.

Finally, although a better outcome was observed with combination therapy compared with adalimumab monotherapy, this difference disappeared after administration of ciprofloxacin was discontinued. We speculate that this might partially be due to the small number of patients of included in the trial, as the loss of significance was solely due to loss of response in some patients in the combination treatment group. On the other hand, as long term treatment with ciprofloxacin for antibioticdependent chronic pouchitis is an established treatment option $^{27}$ without occurrence of $C$ difficile colonisation, ${ }^{28}$ it is tempting to speculate that long term combination treatment of adalimumab with ciprofloxacin results in a more consistent closure of perianal fistulas. Given the fact that the effect of ciprofloxacin is lost after its discontinuation, the better response rate might be at least partially related to a better control of the residual microflora which potentially remains present in the medically closed fistula tract. This hypothesis is corroborated by recent in vitro data revealing that bacterial wall components are involved in fistula pathogenesis, suggesting that antibiotics might contribute to fistula closure. $^{29}$

In conclusion, the ADAFI trial clearly shows that combination therapy of adalimumab with ciprofloxacin is associated with a higher fistula closure rate with a clinically significant impact on HRQoF, IBDQ and CDAI compared with adalimumab monotherapy over a 3-month period. However, after discontinuation of antibiotic therapy, the beneficial effect of initial coadministration is not maintained.

\section{Author affiliations}

${ }^{1}$ Department of Gastroenterology and Hepatology, Erasmus MC Rotterdam, Rotterdam, The Netherlands

${ }^{2}$ Department of Gastroenterology and Hepatology, University Medical Center Utrecht, Utrecht, The Netherlands

${ }^{3}$ Department of Gastroenterology and Hepatology, University Medical Center Leiden, Leiden, The Netherlands

${ }^{4}$ Department of Gastroenterology and Hepatology, University Medical Center Maastricht, Maastricht, The Netherlands

${ }^{5}$ Department of Gastroenterology and Hepatology, Academic Medical Center Amsterdam, Amsterdam, The Netherlands

${ }^{6}$ Department of Gastroenterology and Hepatology, University Medical Center Groningen, Groningen, Groningen, The Netherlands

${ }^{7}$ Department of Gastroenterology and Hepatology, Medisch Spectrum Twente, Enschede, The Netherlands

${ }^{8}$ Department of Gastroenterology and Hepatology, VU University Medical Center Amsterdam, Amsterdam, The Netherlands

Contributors CJVdW, PD and BEH had full access to all data of the study and take responsibility for the integrity of the data and the accuracy of the data analysis. Study concept and design: CJvdW. Acquisition of data: CJvdW, PD, EV, BO, DWH, $M P, C I J P, H M v D, M R$ and AAvB. Analysis and interpretation of data: CJvdW, BEH and PD. Drafting the manuscript: CJvdW, BEH and PD. Critical revision of the manuscript for important intellectual content: CJvdW, PD, EV, BO, DWH, MP, CIJP, $H M v D, M R$ and AAvB. Statistical analysis: BEH. Administrative, technical or material support: The Foundation for Liver and Gastrointestinal Research (SLO).

Funding Financial support was received from Abbott Laboratories Inc. However, the study design, performance, analysis and reporting were conducted without any influence of Abbott Laboratories.

Competing interests All authors state that there are no potential conflicts of interest during the work under consideration for publication. CJW has participated in the advisory board of Centocor, MSD, FALK Benelux, Abbott laboratories and Ferring during the last 3 years and received financial compensation for these activities. AvB has participated in the advisory board of MSD, Abbott laboratories and Vifor during the last 3 years and received financial compensation for these activities. CYP received speakers, consultancy honoraria and unrestricted research grants from Schering Plough, Falk Pharma, Tramedico, Abbott Inc. and Glaxo Smith Kline. DWH and AvB have participated in the advisory board of AbbVie during the last 3 years and received financial compensation for these activities. BO has acted as a consultant for Abbott. MSD and received payment for lectures and manuscript preparation from Ferring.

\section{Patient consent Obtained.}

Ethics approval The protocol was approved by the institutional review board or by the ethics committee at each centre: Erasmus MC, AMC, VUmc, LUMC, UMCU, UMCG, Enschede, MUMC.

Provenance and peer review Not commissioned; externally peer reviewed.

\section{REFERENCES}

1 Lapidus A, Bernell 0, Hellers G, et al. Clinical course of colorectal Crohn's disease: a 35-year follow-up study of 507 patients. Gastroenterology 1998;114:1151-60. 
2 Schwartz DA, Loftus EV Jr, Tremaine WJ, et al. The natural history of fistulizing Crohn's disease in Olmsted County, Minnesota. Gastroenterology 2002:122:875-80.

3 Haapamaki J, Turunen U, Roine RP, et al. Impact of demographic factors, medication and symptoms on disease-specific quality of life in inflammatory bowel disease. Qual Life Res 2009;18:961-9.

4 Schwartz DA, Pemberton JH, Sandborn WJ. Diagnosis and treatment of perianal fistulas in Crohn disease. Ann Intern Med 2001;135:906-18.

5 Bernstein $\mathrm{LH}$, Frank MS, Brandt $\mathrm{L}$, et al. Healing of perineal Crohn's disease with metronidazole. Gastroenterology 1980;79:357-65.

6 Thia KT, Mahadevan U, Feagan BG, et al. Ciprofloxacin or metronidazole for the treatment of perianal fistulas in patients with Crohn's disease: a randomized, double-blind, placebo-controlled pilot study. Inflamm Bowel Dis 2009;15:17-24.

7 Present $\mathrm{DH}$, Rutgeerts $\mathrm{P}$, Targan S, et al. Infliximab for the treatment of fistulas in patients with Crohn's disease. N Engl J Med 1999;340:1398-405.

8 Sands $\mathrm{BE}$, Anderson FH, Bernstein $\mathrm{CN}$, et al. Infliximab maintenance therapy for fistulizing Crohn's disease. N Engl J Med 2004;350:876-85.

9 Colombel JF, Sandborn WJ, Rutgeerts P, et al. Adalimumab for maintenance of clinical response and remission in patients with Crohn's disease: the CHARM trial. Gastroenterology 2007;132:52-65.

10 Colombel JF, Schwartz DA, Sandborn WJ, et al. Adalimumab for the treatment of fistulas in patients with Crohn's disease. Gut 2009;58:940-8.

11 West RL, van der Woude CJ, Hansen BE, et al. Clinical and endosonographic effect of ciprofloxacin on the treatment of perianal fistulae in Crohn's disease with infliximab: a double-blind placebo-controlled study. Aliment Pharmacol Ther 2004:20:1329-36.

12 Hinojosa J, Gomollon F, Garcia S, et al. Efficacy and safety of short-term adalimumab treatment in patients with active Crohn's disease who lost response or showed intolerance to infliximab: a prospective, open-label, multicentre trial. Aliment Pharmacol Ther 2007:25:409-18.

13 Irvine EJ. Usual therapy improves perianal Crohn's disease as measured by a new disease activity index. McMaster IBD Study Group. I Clin Gastroenterol 1995;20:27-32.

14 de Boer AG, Wijker W, Bartelsman JF, et al. Inflammatory bowel disease questionnaire: cross-cultural adaptation and further validation. Eur I Gastroenterol Hepatol 1995;7:1043-50.

15 Irvine EJ, Feagan BG, Wong CJ. Does self-administration of a quality of life index for inflammatory bowel disease change the results? J Clin Epidemiol 1996;49:1177-85.

16 Lichtiger S, Binion DG, Wolf DC, et al. The CHOICE trial: adalimumab demonstrates safety, fistula healing, improved quality of life and increased work productivity in patients with Crohn's disease who failed prior infliximab therapy. Aliment Pharmacol Ther 2010;32:1228-39.

17 Arnold GL, Beaves MR, Pryjdun VO, et al. Preliminary study of ciprofloxacin in active Crohn's disease. Inflamm Bowel Dis 2002;8:10-5.

18 Ciccocioppo R, Bernardo ME, Sgarella A, et al. Autologous bone marrow-derived mesenchymal stromal cells in the treatment of fistulising Crohn's disease. Gut 2011;60:788-98.

19 Irvine EJ, Feagan B, Rochon J, et al. Quality of life: a valid and reliable measure of therapeutic efficacy in the treatment of inflammatory bowel disease. Canadian Crohn's Relapse Prevention Trial Study Group. Gastroenterology 1994;106:287-96.

20 Vogelaar L, Spijker AV, van der Woude CJ. The impact of biologics on health-related quality of life in patients with inflammatory bowel disease. Clin Exp Gastroenterol 2009;2:101-9.

21 Bassler D, Montori VM, Briel M, et al. Early stopping of randomized clinical trials for overt efficacy is problematic. J Clin Epidemiol 2008;61:241-6.

22 van Bodegraven AA, Sloots CE, Felt-Bersma RJ, et al. Endosonographic evidence of persistence of Crohn's disease-associated fistulas after infliximab treatment, irrespective of clinical response. Dis Colon Rectum 2002;45:39-45; discussion -6.

23 Van Assche G, Vanbeckevoort D, Bielen D, et al. Magnetic resonance imaging of the effects of infliximab on perianal fistulizing Crohn's disease. Am J Gastroenterol 2003:98:332-9.

$24 \mathrm{Ng} \mathrm{SC}$, Plamondon S, Gupta A, et al. Prospective evaluation of anti-tumor necrosis factor therapy guided by magnetic resonance imaging for Crohn's perineal fistulas. Am J Gastroenterol 2009;104:2973-86.

25 Tozer P, Ng SC, Siddiqui MR, et al. Long-term MRI-guided combined anti-TNF-alpha and thiopurine therapy for crohn's perianal fistulas. Inflamm Bowel Dis 2012;18:1825-34.

26 Karmiris K, Bielen D, Vanbeckevoort D, et al. Long-term monitoring of infliximab therapy for perianal fistulizing Crohn's disease by using magnetic resonance imaging. Clin Gastroenterol Hepatol 2011;9:130-6.

27 Pardi DS, Sandborn WJ. Systematic review: the management of pouchitis. Aliment Pharmacol Ther 2006;23:1087-96.

28 McLaughlin SD, Clark SK, Tekkis PP, et al. An open study of maintenance antibiotic therapy for chronic antibiotic-dependent pouchitis: efficacy, complications and outcome. Colorectal Dis 2011;13:438-44.

29 Scharl M, Frei P, Pesch T, et al. OP20 TNF and MDP induce epithelial-tomesenchymal transition in human intestinal cells: Implications for the pathogenesis of Crohn's disease-associated fistulae and the use of anti-TNF antibodies. J Crohns Colitis 2012;6:S10. 


\section{GUT}

\section{Adalimumab combined with ciprofloxacin is superior to adalimumab monotherapy in perianal fistula closure in Crohn's disease: a randomised, double-blind, placebo controlled trial (ADAFI)}

Pieter Dewint, Bettina E Hansen, Elke Verhey, et al.

Gut 2014 63: 292-299 originally published online March 23, 2013 doi: 10.1136/gutjnl-2013-304488

Updated information and services can be found at:

http://gut.bmj.com/content/63/2/292.full.html

These include:

References This article cites 29 articles, 2 of which can be accessed free at: http://gut.bmj.com/content/63/2/292.full.html\#ref-list-1

Email alerting Receive free email alerts when new articles cite this article. Sign up in service the box at the top right corner of the online article.

Collections

Articles on similar topics can be found in the following collections

Crohn's disease (868 articles)

Notes

To request permissions go to:

http://group.bmj.com/group/rights-licensing/permissions

To order reprints go to:

http://journals.bmj.com/cgi/reprintform

To subscribe to BMJ go to:

http://group.bmj.com/subscribe/ 